\title{
A Survey of Feature Base Methods for Human Face Detection
}

\author{
Hiyam Hatem ${ }^{1,2}$, Zou Beiji ${ }^{1}$ and Raed Majeed ${ }^{1}$ \\ ${ }^{1}$ School of Information Science and Engineering, Central South University, \\ Changsha, Hunan 410083, China, \\ ${ }^{2}$ Department Of Computer Science, Collage of Sciences, Baghdad University, \\ Iraq \\ hiamhatim2005@yahoo.com,bjzou@vip.163.com, \\ raed.m.muttasher@gmail.com
}

\begin{abstract}
The human face is among the most significant objects in an image or video, it contains many important information and specifications, also is required to be the cause of almost all achievable look variants caused by changes in scale, location, orientation, pose, facial expression, lighting conditions and partial occlusions. It plays a key role in face recognition systems and many other face analysis applications.

We focus on the feature based approach because it gave great results on detect the human face. Face feature detection techniques can be mainly divided into two kinds of approaches are Feature base and image base approach. Feature base approach tries to extract features and match it against the knowledge of the facial features.

This paper gives the idea about challenging problems in the field of human face analysis and as such, as it has achieved a great attention over the last few years because of its many applications in various domains. Furthermore, several existing face detection approaches are analyzed and discussed and attempt to give the issues regarding key technologies of feature base methods, we had gone direct comparisons of the method's performance are made where possible and the advantages/ disadvantages of different approaches are discussed.
\end{abstract}

Keywords: Facial Features, Viola-Jones Feature, Skin Color Detection

\section{Introduction}

Digital images and video are becoming more and more important in the multimedia information era. Object detection is one of the computer technologies, which is connected to the image processing and computer vision and it interacts along detecting instances of objects of the specified class, such as human faces, building, tree, car etc. The objects can be taken from the digital images or video frames.

Now days a face takes on the major function in sociable intercourse with regard to conveying id and also the feelings of the person. Persons have a marvelous ability to identify different faces than machines. Therefore, face detection plays major role in face recognition, facial expression recognition, head-pose estimation, human-computer interaction, etc. Face detection is a computer technology that determines the location and size of human faces in arbitrary (digital) image.

The human face is one of the most important objects in an image or video. It's an active area of research in pattern recognition and image processing. Its wide range of practical applications includes personal identity verification, video-surveillance, facial expression extraction, advanced human and computer interaction, computer vision etc.

Face detection and tracking is a rapidly rising analysis spot because of rising demands for security in commercial and law enforcement applications. Demands and research 
activities in machine recognition of human faces from still and video images have increased significantly over the past 30 years [1].

During the past several years, the face detection problem has been given a significant focus due to the range of its applications in commerce and law enforcement. Moreover, in recent years a lot of pattern recognition and heuristic based methods have been proposed for detecting human face in images and videos [2]. Face detection is the first stage of many face processing systems, including face recognition, automatic focusing on cameras, automatic face confusion in pictures, pedestrian and driver drowsiness detection in cars, criminal identification, access control, etc. [3].

Facial expression detection/recognition from images and video sequences is an active research area. Analysis of facial expressions by the machine is a challenging task with many applications. Computer vision techniques are already given to the understanding of facial expression detection. Such applications have been reported in Lekshmi et al. [4] For face detection, [5] for facial feature extraction and [6] for face recognition.

With increasing terrorist pursuits and augmenting the demand for video surveillance, it was the need of an hour to come up with an efficient and fast detection and tracking algorithm. This detecting and tracking algorithm will bring practical applications like Smart captcha, Webcam based energy/power saver, Time tracking service, Outdoor surveillance camera service, Video chats service, Teleconferencing [7].

\section{Statement of the Problem}

Several applications, such as face processing, computer, human interaction, human crowd surveillance, biometric, video surveillance, artificial intelligence and content-based image retrieval etc. All of these applications, stated above, require face detection, which is often simply considered as a preprocessing step, for obtaining the "object". In other words, many of the techniques are proposed for these applications assume that, the location of the face is pre-identified and available for the next step.

There are many problems associated with face detection and it is one of the challenging problems in image processing due to building systems that perform facial recognition. It is essential in applications such as video surveillance, human computer interface and face recognition. The first problem is coming in the way of face detection, is chosen proper color model for skin color segmentation. There are several color models and each has specific work field and strength. Facial detection depends on the characteristic of the acquired image and can be very sensitive to noise and poor lighting conditions. The challenges associated with face detection can be attributed to the following factors:

- Pose: - In a surveillance system, the camera is mostly mounted in a location where the people are unable to attain to the camera. Mounting a camera a high location, the faces are viewed by some angle degree. This is the simplest case with city surveillance applications. The next and the most challenging situations is that people naturally pass through the camera view. They do not even look at the camera lens. Authorities cannot restrict the person's behaviors in public places. Furthermore the images of a face vary due to the relative camera-face pose (frontal, 45 degrees, profile, upside down), and some facial features such as an eye or the nose may come to be partially or totally occluded.

- Facial expression: - Facial Expression is one of the most influential, temperaments, and immediate signifies for human beings to converse their emotions and meanings. The Facial expression is related to the appearance of face like angriness or happiness which can directly affect the individual's face. The appearance of a person who is laughing is totally different than the appearance of a person who is angry. Therefore, facial expressions directly affect the appearance of the face in the image.

- Occlusion: - Faces some time occluded by other objects. In an image with a group of people, some faces may partially occlude other faces. Occlusion is the obstructing the 
face(s) in images that can be covered by part or the whole of other objects. For instance, a face in an image can be partially or fully covered with other peoples' faces.

- Image orientation: - Image orientation depends on the nature of the images may appear correct, upside- down, rotated, or inversed from left to right and it looks like trying to read a sign in a mirror. Face images directly vary for different rotations about the camera's optical axis.

- Imaging conditions: - When the image is formed, factors such as lighting (spectra, source distribution and intensity) and camera characteristics (sensor response, lenses) affect the appearance of a face.

- Different facial features: - A lot of people wear glasses, some have a beard or a mustache, others have a scar. These types of features are called facial features, there are many cases of facial features and they all vary in shape, size and color.

- Face size: - The size of the human face can vary a lot. Not only do different persons have different sized faces, also faces closer to the camera appear larger than faces that are far away from the camera.

- Illumination: - Illumination is an important factor in determining the quality of images and also can have much effect on the evaluation of the image and consequently detected faces. The factor is related to the lighting and the angle of light that exist in the images. Faces seem different when different lighting conditions are used. For instance, when side lighting is used, a part of the face is very bright while the other part is very dark.

\section{Face Detection Techniques}

Human face detection means that for a given image or video, to determine whether it includes face regions, if so, determines the number, the exact location and the size of all the faces. The performance of various faces based applications, from traditional face recognition and verification to the modern face clustering, tagging and retrieval, relies on accurate and efficient face detection [8]. The ability to detect faces in a scene is important for humans in their everyday activities. Consequently, automating this could well be practicing in numerous application areas such as intelligent human-computer interfaces, content-based image retrieval, security, surveillance, gaze-based control, video conferencing, speech recognition assistance, video compression as well as many other areas.

The goal of face detection is to determine if there are any faces in the image or not and, if present, return the location and the bounding box of each face in the image. Human faces are difficult to model as it is crucial to are the cause of all probable appearance variations attributable to changes throughout the scale, location, orientation, facial expression, lighting conditions and partial occlusions, etc. [9]. The result of detection gives the face location parameters and it could be required in various forms, for instance a rectangle covering the central part of the face, eye centers or landmarks including eyes, nose and mouth corners, eyebrows, nostrils, etc.

Feature based methods have some advantages which are rotation independency, scale independence, and their execution time are so quick, in comparing to other methods [10]. Feature based methods contain facial features, skin color, texture, and multiple features. Basically, there are two kinds of approaches to detect facial part in the given image i.e. Feature base and image base approach. Feature base approach tries to extract features of the image and match it against the knowledge of the facial features. Although the image base approach tries to get the best match between training and testing images. 


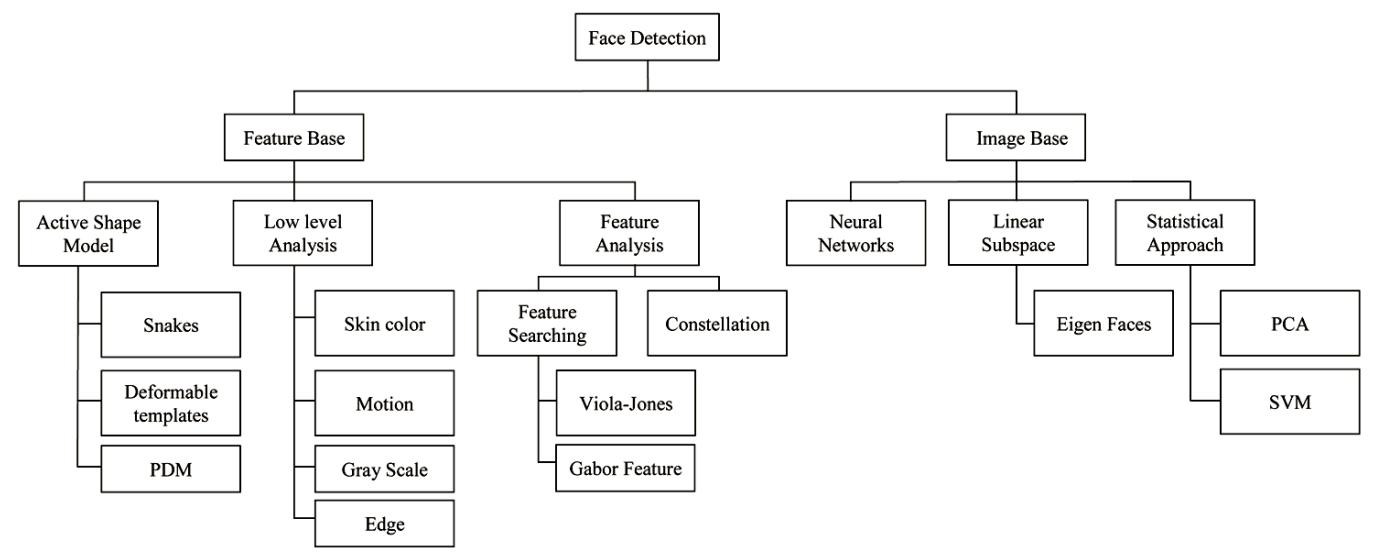

Figure 1. General Face Detection Methods

\section{Feature Base Approach}

Objects are usually recognized by their unique features. There are many features in human face, which can be recognized between a face and many other objects. It locates faces by extracting structural features like eyes, nose, mouth etc. and then uses them to detect a face. Typically, some sort of statistical classifier qualified then helpful to separate between facial and non-facial regions. Many feature extraction methods have been proposed in the literature. The problem with these algorithms is that these features are corrupted due to illumination, occlusion and noise.

Furthermore, some studies have proven that color of skin is an excellent feature for detecting faces among other objects due to different people have different skin color and it is more clear when the race of people is also a metric of evaluation [11]. In addition, human faces have particular textures which can be used to differentiate between face and other objects. Moreover, edge of features can help to detect the objects from the face. In addition, using blobs and streaks can assist to discover objects from a given image. Feature based methods have some advantages which are rotation independency, scale independence, and their execution time are so quick, in comparing to other methods [10]. Hjemal and Low [12] further divide this technique into three categories: low level analysis, feature analysis and active shape model.

\subsection{Active Shape Model}

Active shape models (ASMs) are statistical models of the shape of the objects as constrained by the point distribution model, the shape of an object is reduced to a set of points. This technique has been widely used to analyze facial images, mechanical assemblies and 2D and 3D medical images. These are used to define then actual physical and higher-level appearance of features. These models are released near to a feature, such that they interact with the local image, deforming to take the shape of the feature [12]. ASM are models of the shapes of objects which iteratively deform to fit to an example of the object in a new image. It works in following two steps: Look at the image around each point for a better position for that point, update the model parameters to best match to these new found positions.

Active shape models focus on complex non-rigid features like actual physical and higher level appearance of features [13]. ASMs are utilized successfully in many application areas, including face recognition $[14,15]$, industrial inspection and medical image interpretation. However, ASMs only use data around the model points, and do not take advantage of all the gray-level information available across an object. Means that Active Shape Models (ASMs) tend to be directed at on auto-pilot locating landmark 
points that define the shape of any statistically modeled object in an image, when of facial features such as the eyes, lips, nose, mouth and eyebrows. The training stage of an ASM contains the building of a statistical facial model from a training set containing images with manually annotated landmarks.

ASMs is classified into three groups i.e. Snakes, PDM, definable templates. Using a dimensionality reduction technique such as PCA on this data results in an Active Shape Model (ASM) [16], capable of representing the primary modes of shape variation. Simply by looking at the largest principal components, one can find the directions in the data that match the versions in pitch and yaw. If the location of the facial features were known in a new image, pose could be estimated by projecting the feature locations into the shape subspace and assessing your elements in charge of posing [17]. In [18] present a method for mapping the Peking Opera facial makeup onto a frontal human face in an image based on modified Active Shape Model (ASM), Delaunay triangulation and affine transformation.

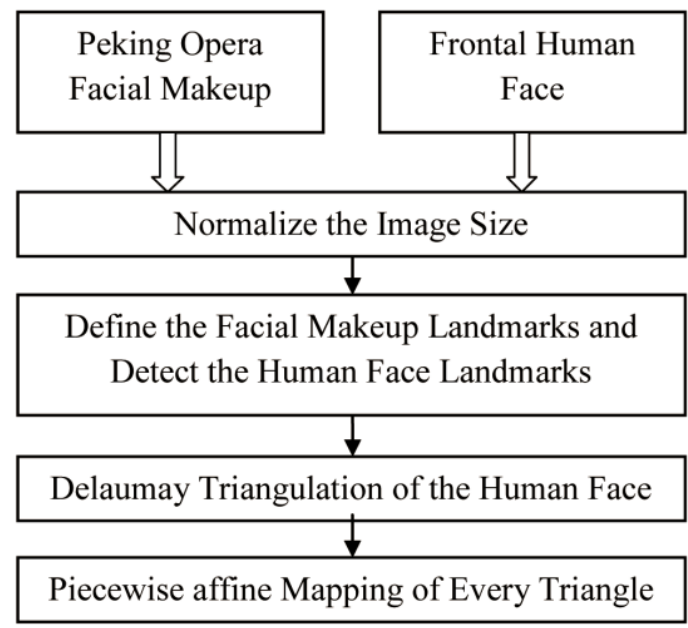

Figure 2. Flow Chart of the Mapping Implementation

In the 2D data domain, Active Shape Model (ASM) [16], Active Appearance Model (AAM) [19] and more recently, Active Orientation Model (AOM) [20] have been proposed. The ASM approach builds 2D shape models and uses their constraints along with some information on the image content near the 2D shape landmarks to locate points on new images. In [21] the problem is addressed employing Active Shape Models (ASM) structured with a Support Vector Machine (SVM) classifier. They define four ratios from features in the human face, using FACS Action Units to classify emotions. These are snakes, deformable templates, and point distribution models will describe as follows:

4.1.1. Snakes: In this approach, active contours or snakes are used to locate head boundary. In addition, features 'boundaries can be found by these contours. To achieve our task we have to initialize the starting position of the snake, which may be in the proximity around the head boundary.

4.1.2. Deformable Templates: Locating facial feature boundaries by using active contours is not an easy task. Finding and locating facial edges is difficult. Sometimes there can be edge detection problems because of bad lighting or bad contrast of images. Therefore, we need methods that are more flexible. Deformable templates, approaches are developed to solve this problem; Deformation is based on local valley, edge, peak, and brightness. Other than face boundary, the salient feature (eyes, nose, mouth and eyebrows) extraction is a great challenge of face recognition. In this method some 
predefined templates are used to guide the detection process. These predefined templates are very flexible and able to change their size and other parameter values to match themselves to the data. The final values of these parameters can be used to describe the features.

4.1.3. Point Distribution Models: These models are compact parameterized descriptions of the shapes based on statistics. The implementation process of PDM is quite different from the other active shape models. The contour of PDM is discredited into a set of labeled points. Now, the variations of these points can be parameterized over a training set that that includes objects of different sizes and poses. We can construct these variations of features as a linear flexible model [22].

A mixture model of factor analyzers has recently been extended [23] and applied to face recognition [24]. Both studies show that FA perform better than PCA in digit and face recognition. Since pose, orientation, expression, and lighting affect the appearance of a human face, the syndication of faces in the image space can be better showed by a multimodal density model where each modality captures certain characteristics of certain face appearances. They provide a probabilistic method that uses a mixture of factor analyzers (MFA) to detect faces with wide variations, the parameters in the mixture model are approximated applying an EM algorithm.

In the proposed distribution-based face detection [25, 26], In the first step, the face likelihood distribution is generated from an input scene, they can calculate the face likelihood using the calibrated classifier because the face likelihood is the posterior possibility when the output class is facing. The key is that a true face, even if a small warp is applied, still has a high face likelihood, in other words, the high face likelihood region develops about an authentic face. In contrast, non-faces with high face likelihood tend to appear at points, not regions, in addition, if we binaries the face likelihood by threshold in distribution procedure, the process will equal to the sub window-based procedure. In other words, the proposed distribution-based face detection is a generalized version of the sub window-based face detection. Each position of the face likelihood has the face likelihood of the equivalent sub window. The distribution has three-dimensions: horizontal, vertical, and scale. Clear differences exist between the face likelihood distribution around faces and non-faces. This difference can provide useful information to classify the falsely detected non-face correctly [27].

Sung and Poggio developed a distribution-based system for face detection [28,29] which shown how the distributions of image patterns from one object class can be learned from positive and negative examples (i.e., images) of that class. Their system consists of two components, distribution-based models for face/nonface patterns and a multilayer perception classifier. Each face and nonface example, are first normalized and processed into a 19x19 pixel image and treated as a 361-dimensional vector or pattern. Next, the patterns are grouped into six faces and six nonface clusters using a modified k-means algorithm. The system designed by Sung and Poggio consists of the following four steps [30]:

1. First the image in the detection window is preprocessed by recalling it to $19 \times 19$ pixels. This preprocessing step enhances the image and reduces the dimensionality of the image vector from $<361$ to $<283$.

2. By using 12 multi-dimensional Gaussian clusters a distribution-model of canonical face- and nonface patterns is constructed. The 283-dimensional clusters are produced using a modified k-means clustering algorithm which computes the cluster centroid and covariance matrices.

3. Given a new image, the distance between that image pattern and each cluster is computed, resulting in 12 distances between the image and the 12 cluster centroid. The first value is the Mahalanobis-like distance between the new image and the cluster centroid in a subspace spanned by the cluster's 75 largest eigenvectors. The second 
value is the Euclidean distance between the new image and its projection in the subspace. Given 12 distances and two values per distance, in total a 24-dimensional image measurement vector is obtained.

4. A multilayer perception (MLP) is used to classify input patterns as faces or non-faces, using the 24-dimensional image measurement vector. The MLP is trained using standard back propagation from a training set [30].

\subsection{Low Level Analysis}

Low level analysis first dealt with segmentation of visual features using pixel properties such as edge detection, gray scale analysis, color information. Features generated from low-level analysis are likely to be ambiguous. For instance, in locating facial regions using a skin color model, background objects of similar color can also be detected. In [12] implemented an edge representation method for detecting the facial features in line drawings by detecting the changes in pixel properties. They developed this further to detect the human head outline. The edge based techniques rely upon the labeled edges which are matched to a face model for verification. Generally eyebrows, pupils and lips appear darker than surrounding regions, and thus extraction algorithms can search for local minima. In contrast, local maxima can be used to indicate the bright facial spots such as nose tips. Detection is then performed using low-level grayscale thresholding [31].Based on low-level visual features like color, intensity, edges, motion etc.

4.2.1. Skin Color Base: Color is a vital feature of human faces. Using skin-color as a feature for tracking a face has several advantages. Color processing is much faster than processing other facial features. Under certain lighting conditions, color is orientation invariant. This property makes motion estimation less of a challenge since just a interpretation model should be applied for motion estimation [32]. There are a number of skin color model like:

i. RGB Color Space: It is an additive color model in which red, green, and blue light are added together in various ways to reproduce a broad array of colors. The main purpose of the RGB color model is for the sensing, representation, and display of images in electronic systems, such as televisions and computers, though it has also been used in conventional photography. Before the electronic age, the RGB color model already had a solid theory behind it, based on human perception of colors [33]. The normalized RGB color space can be calculated using the original RGB components as following:

$$
\begin{aligned}
& r=\frac{R}{R+G+B} \\
& g=\frac{G}{R+G+B} \\
& b=\frac{B}{R+G+B}
\end{aligned}
$$

ii. $\mathbf{Y C}_{\mathbf{b}} \mathbf{C}_{\mathbf{r}}$ Color Space: $\mathrm{YC}_{\mathrm{b}} \mathrm{C}_{\mathrm{r}}$ is a family of color spaces used as a part of the color image in video and digital photography systems. $\mathrm{Y}$ is the luma component and $\mathrm{C}_{\mathrm{b}}$ and $\mathrm{Cr}$ are the blue-difference and red-difference chroma components. $\mathrm{Y}$ (with prime) is distinguished from $\mathrm{Y}$ which is luminance, meaning that light intensity. The luminance component $(\mathrm{Y})$ of $\mathrm{YC}_{\mathrm{b}} \mathrm{Cr}$ is independent of the color, so can be adopted to solve the illumination variation problem and it is easy to program.

iii. HSV Color Space: Since hue, saturation and intensity value are three properties used to describe color, it seems logical that there is a corresponding color model. Essentially, HSV-type color spaces are deformations of the RGB color cube and they can be mapped from the RGB space via a nonlinear transformation. One of the advantages of 
these color spaces in skin detection is that they allow users to intuitively specify the boundary of the skin color class in terms of the hue and saturation.

In [34] propose a skin color based face detection technique. First, the input image is resized and light corrected. Then, the resulted image is segmented based on skin color, using a combination of segmentation in RGB and HSV color spaces, followed by segmentation in $\mathrm{YCbCr}$ using the Elliptical model. Tracking human faces using color as a feature has several problems like the color representation of a face obtained by a camera is influenced by many factors (ambient light, object movement, etc.).

Majorly three different face detection algorithms are available based on $\mathrm{RGB}, \mathrm{YCbCr}$, and HIS color space models. In the implementation of the algorithms, there are three main steps viz. Crowley and Coutaz [35] suggested simplest skin color algorithms for detecting skin pixels. The perceived human color varies as a function of the relative direction of the illumination. The pixels in skin region can be detected using a normalized color histogram, and can be normalized for changes in intensity on dividing by luminance. Converted a $[R, G, B]$ vector is converted into a $[r, g]$ vector of normalized color which provides a fast means of skin detection. This algorithm fails when there is some more skin region like legs, arms, etc. Cahi and Ngan [36] suggested skin color classification algorithm with $\mathrm{YCbCr}$ color space.

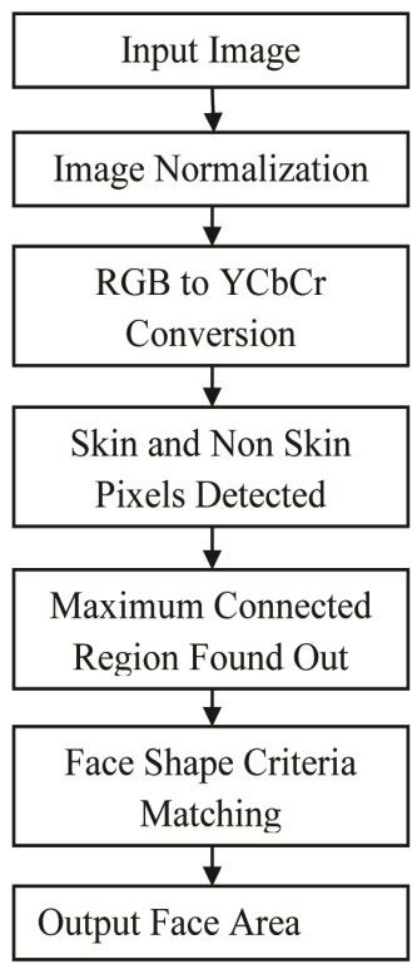

Figure 3. Sample of Skin Color Detection Algorithm [36]

4.2.2. Motion Base: When use of video sequence is available, the motion information can be used to locate moving objects. Going silhouettes like face and body parts can be extracted by simply threading accumulated frame differences [37]. Besides face regions, facial features can be located by frame differences [38]. Yao and Cham [39] propose an efficient method that estimates the motion parameters of a human head from a video sequence by using a three-layer linear iterative process.

In [40] they address two issues concerning real-world time-continuous emotion detection from videos of users' faces: (i) the impact of weak ground truth on the emotion detection accuracy and (ii) the impact of the users' facial expression on the emotion detection accuracy. They implemented an appearance-based emotion detection algorithm 
that uses Gabor features and a $\mathrm{k}$ nearest neighbor classifier. The emotion detection procedure involved three stages: (i) pre-processing, (ii) low level feature extraction, and (iii) emotion detection.

4.2.3. Gray Scale Base: Gray information within a face can also be treated as important features. Facial features such as eyebrows, pupils, and lips appear generally darker than their surrounding facial regions. Several of the latest feature extraction algorithms [41] search for local gray minima within segmented facial regions. In these algorithms, the input images are first enhanced by contrast-stretching and grayscale morphological routines improve the quality of local dark patches and thereby make detection easier. The extraction of dark patches is achieved by low-level grayscale thresholding.

4.2.4. Edge Base: The edge based techniques rely upon the labeled edges which are matched to a face model for verification. Generally eyebrows, pupils and lips appear darker than surrounding regions, and thus extraction algorithms can search for local minima [31] .Edge is the most ancient feature in computer vision applications and it was applied in some earlier face detection techniques like Sakai et al. [42]. This work was based on analyzing line drawings of the faces from photographs, aiming to locate facial features. Than later Craw et al. [43] proposed a hierarchical framework based on Sakai i work to trace a human head outline. Then after remarkable works were carried out by many researchers in this specific area.

The method suggested by Anila and Devarajan [44] was very simple and fast. They proposed framework which consists three steps i.e. Initially the images are enhanced by applying a median filter for noise removal and histogram equalization for contrast adjustment. In the second step the edge image is constructed from the enhanced image by applying the operator. Then a novel edge tracking algorithm is applied to extract the sub windows from the enhanced image based on edges. Further, they used Back propagation Neural Network (BPN) algorithm to classify the sub-window as either face or non-face [41].

\subsection{Feature Analysis}

These algorithms aim to find structural features that exist even when the pose, viewpoint, or lighting conditions vary, and then use these to locate faces. The feature analysis is based on both the knowledge of an adequate face model (prior model) and on the proportions of normalized distances and angles derived from the individual description of face parts (eyes, nose, mouth).It uses additional knowledge about the face and removes the ambiguity produces by low level analysis. The first involves sequential feature searching strategies based on the relative positions of individual facial features [31]. Initially prominent facial features are determined which allows less prominent features to be hypothesized. With this first family of methods, processing is potentially fast as no learning base is necessary. The methods for parameter extraction are often specific to the context at hand, and are constructed empirically with color, edge or motion cues [45]. These methods are designed mainly for face localization.

4.3.1 Feature Searching: Feature searching techniques begin with the determination of prominent facial features. The detection of the prominent features, then allows for the existence of other less prominent features to be hypothesized using anthropometric measurements of facial geometry. The detection of the prominent features, then allows for the existence of other less prominent features to be hypothesized using anthropometric proportions of facial geometry. Among the literature survey, a pair of eyes is the most generally employed reference features due to its distinct side-by-side appearance. Other features include a main face axis, outline (top of the head) and body (below the head). Jeng et al. [46] propose a system for face and facial feature detection, which is also based 
on anthropometric measures. In their system, they initially try to establish achievable locations of the eyes in binarized pre-processed images. For each possible eye pair the algorithm goes on to search for a nose, a mouth, and eyebrows. Each facial feature has an affiliated evaluation function, which is used to determine the final most likely face candidate, weighted by their facial importance with manually selected coefficients. It contains the Viola-Jones method and Gabor feature method will describe as follows:

* Viola-Jones Method: The first object detection framework to provide competitive object detection rates in real-time proposed in 2001 by Paul Viola and Michael Jones [47]. Although it can be trained to detect a variety of object classes, it was motivated primarily by the problem of face detection. The Viola and Jones, the University of Cambridge's students, proposed the first real-time face detection method, Cascade classifier method. There are two main factors that determine the effectiveness of an FD system: the system's detection accuracy and its processing speed. Although the detection accuracy has been improved through many novel approaches during the last ten years, the speed is still a problem impeding the wide use of the FD system in real time applications. One of the biggest steps toward improving the processing speed and making the real time implementation possible has been the introduction of the AdaBoost and cascade FD, proposed by Viola and Jones [47, 26]. In this chapter, the basics of AdaBoost and cascade algorithms, their extensions and available implementations are briefly described. It is a state-of-the-art face detection model that provides outstanding computational efficiency. It contains three main ideas that make it possible to build a successful face detector that can run in real time: the integral image, classifier learning with AdaBoost, and the attentional cascade structure. Cascade classifier method is a face detection algorithm based on Haar-like features. The white region's pixel value sum subtracts the black region's pixel value sum can get the so called characteristic value of feature rectangle, which is used as the face detection's basis.

The amazing real-time speed and high detection accuracy of Viola and Jones' face detector can be attributed to three factors: the integral image representation, the cascade framework, and the use of Adaboost to train cascade nodes. The integral image representation enables the calculation of Haar-like features extremely fast. The cascade framework allows non-face background patches to be filtered away quickly. The Adaboost algorithm selects Haar-like features and combines them into an ensemble classifier in a cascade mode. The integral image and cascade framework make the detector run fast, whereas Adaboost is the key to obtain a high detection rate for a cascade node. The basics of integrated image processing, Haar features and the face detection process are also given. A Haar-like feature can defined as the difference of the sum of pixels in two or more adjacent rectangular regions. By changing the position, size, shape and arrangement of these rectangular regions, Haar-like features can capture the intensity gradient at different locations, spatial frequencies and directions [48].

These face detection procedures classify images based on the value of simple features. There are many motivations for using features rather than the pixels directly. The most common reason is that features can act to encode ad-hoc domain knowledge that is difficult to learn using a finite quantity of training data. In this system, there is also a second critical motivation for features: the feature-based system operates much faster than a pixel-based system. More specifically, there are three kinds of features. The value of a two-rectangle feature is the difference between the sum of the pixels within two rectangular regions. The regions have the same size and shape and are horizontally or vertically adjacent (see Figure 4). A three-rectangle feature computes the sum within two outside rectangles subtracted from the sum in a center rectangle. Finally a four-rectangle feature computes the difference between diagonal pairs of rectangles. 


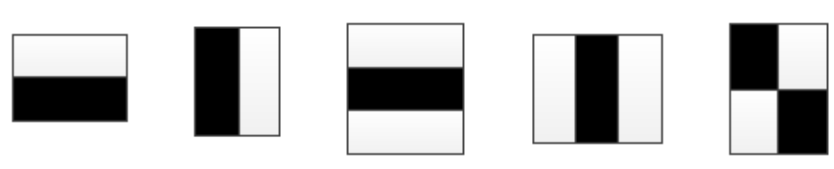

Figure 4. Haar-like Feature Rectangle

A. Integral Image: Haar-like features are digital imaging features used in object recognition. They owe their name to their intuitive similarity with Haar wavelets and were used in the first real-time face detector.[1], it's working with only image intensities (i.e., The RGB pixel values at each and every pixel of the image) made the task of feature calculation computationally expensive. A publication by Papageorgiou et al. [49] discussed working with an alternate feature set based on Haar wavelets instead of the usual image intensities. Viola and Jones [47] adapted the idea of using Haar wavelets and developed the so-called Haar-like features. A Haar-like feature considers adjacent rectangular regions at a specific location in a detection window, sums up the pixel intensities in each region and calculates the difference between these sums. This difference is then used to categorize subsections of an image. For example, let us say we have an image database with human faces. It is a common observation that among all faces the region of the eyes is darker than the region of the cheeks. Therefore a common Haar feature for face detection is a set of two adjacent rectangles that lie above the eye and the cheek region. The position of these rectangles is defined relative to a detection window that acts like a bounding box of the target object (the face in this case).

Rectangle features can be computed very rapidly using an intermediate representation for the image which we call the integral image. The integral image at location $x, y$ contains the sum of the pixels above and to the left of $x, y$, inclusive:

$$
i i(x, y)=\sum_{x^{\prime} \leq x, y^{\prime} \leq y} i\left(x^{\prime}, y^{\prime}\right)
$$

where $i i(x, y)$ is the integral image and $i(x, y)$ is the original image. Using the following pair of recurrences:

$$
\begin{array}{r}
s(x, y)=s(x, y-1)+i(x, y) \\
i i(x, y)=i i(x-1, y)+s(x, y)
\end{array}
$$

Where $s(x, y)$ is the cumulative row sum, $s(x,-1)=0$, and $i i(-1, y)=0)$ the integral image can be computed in one pass over the original image. Using the integral image any rectangular sum can be computed in four array references. Clearly, the difference between two rectangular sums can be computed in eight references. Since the two-rectangle features defined above involve adjacent rectangular sums, they can be computed in six array references, eight in the case of the three-rectangle features, and nine for fourrectangle features.

The brilliance in using an integral image to speed up the feature extraction lies in the fact that any rectangle in an image can be calculated from that image's integral image, in only four indexes to the integral image while the calculation of the integral image itself is done in only one pass of the image [50].

B. Adaboost Core Method: Adaptive Boosting (AdaBoost) [51], is a machine learning algorithm, first formulated by Freund and Schapire [52]. Adaboost algorithm is an iterative algorithm. The key is to train different classifiers (especially weak classifiers) for the same training set, and then congregate these weak classifiers to form a stronger 
final classifier it the strong classification. The algorithm is achieved by changing the data distribution. This is realized by the classification correctness of each sample as well as the accuracy of last time overall classification to determine the weight of each sample. The new data set whose weights are modified will be sent to the lower classifiers for training. Finally, every classifier obtained after training will be integrated as the final decision classifier. It is a meta-algorithm, and can be used in conjunction with many other learning algorithms to improve their performance [53].

AdaBoost is adaptive in the sense that subsequent classifiers built are tweaked in favor of those instances misclassified by previous classifiers. AdaBoost is sensitive to noisy data and outliers. Otherwise, it is less susceptible to the over fitting problem than most learning algorithms. AdaBoost calls a weak classifier repeatedly in a series of rounds $t=$ $1,2, \ldots$ T. For each call, a distribution of weights $D_{t}$ is updated to indicate the importance of examples in the data set for the classification. On each round, the weights of each incorrectly classified example, are increased (or alternatively, the weights of each correctly classified example, are decreased), so that the new classifier focuses more on those examples.

In the FD training algorithm, AdaBoost allows the designer to combine weak and simple learners to form an accurate and complex overall classifier; The following description of AdaBoost is based on the works [51] and [52]. Also, note that the first feature selected by AdaBoost is not symmetric as may be expected from the left-right symmetry of the human face. This is due to asymmetries in the random set of background images that form the non-face training set. Viola and Jones adapted the Discrete AdaBoost algorithm by constraining weaker classifier to use a single filter which best separates the positive and negative examples. For each filter an optimal threshold value is determined such that the minimum number of examples is misclassified. In this particular example the weighting is modified slightly and this is done in order to favor the classification of the face class. Where the deviation from the classical implementation is shown by setting the initial weights to one over the database size, the weighting is set such that the face examples will have a higher weight or importance. This is shown as an initial step in the algorithm. The core of the idea behind the use of AdaBoost is the application of a weight distribution to the sample set and the modification of the distribution during each iteration of the algorithm, where the weights are first normalized and then adjusted. At the beginning, the weight distribution is flat, but after each iteration of the algorithm, each of the weak learner returns a hypothesis and the weight distribution is modified [50].

C. The Attention Cascade: In theory, Adaboost can produce a single committee of decision stumps that generalizes well. However, to achieve that, an enormous negative training set is needed at the outset to gather all possible negative patterns. In addition, a single committee implies that all the windows inside an image have to go through the same lengthy decision process. There has to be another more cost-efficient way. A cascade is employed to reduce image processing time by focusing attention on the more interesting regions of the image. For example the flat regions of an image, clearly do not contain faces and can be quickly discarded by use of a template consisting of only a small number of features. Such a scheme has the potential to greatly improve the speed of the detector, but still allow a large number of features to be evaluated on highly textured regions that may contain faces. The overall process of classifying a sub-window, thus forms a degenerate decision tree, which was called a "cascade" in [47]. As shown in Figure 5, the input sub-windows pass a series of nodes during detection. Each node will make a binary decision whether the window will be kept for the next round or rejected immediately. The number of weak classifiers in the nodes usually increases as the number of nodes a sub-window passes. This is intuitive, since each node is trying to reject a certain amount of negative windows while keeping all the positive examples, and the task 
becomes harder at late stages. Having fewer weak classifiers at early stages also improves the speed of the detector [54].

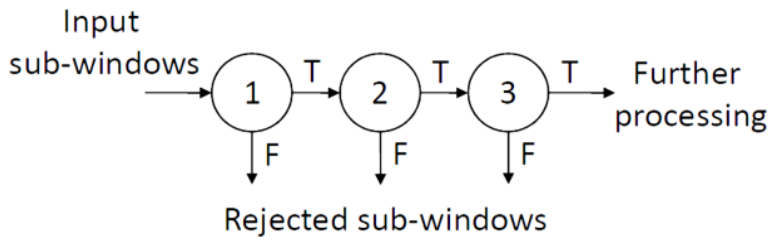

Figure 5. The Attentional Cascade

The cascade structure also has an impact on the training process. Face detection is a rare event detection task. Consequently, there are usually billions of negative examples needed in order to train a high performance face detector. That is, at each node, a threshold was manually chosen, and the partial classifier was used to scan the negative example set to find more un-rejected negative examples for the training of the next node. Furthermore, each node is trained independently, as if the previous nodes do not exist. One argument behind such a process is to force the addition of some nonlinearity in the training process, which could improve the overall performance. However, recent works showed that it is actually beneficial not to completely separate the training process of different nodes. In [55], the attentional cascade is constructed manually. That is, the number of weak classifiers and the decision threshold for early rejection at each node are both specified manually. This is a non-trivial task. If the decision thresholds were set too aggressively, the final detector will be very fast, but the overall detection rate may be hurt. On the other hand, if the decision thresholds were set very conservatively, most subwindows will need to pass through many nodes, making the detector very slow. Combined with the limited computational resources available in early 2000 's, it is no wonder that training a good face detector can take months of fine-tuning [54].

4.3.2. Constellation Method: Constellations are then formed only from candidates that lie inside the appropriate locations, and the most face-like constellation is determined. Finding the best constellation is formulated as a random graph matching problem in which the nodes of the graph correspond to features of a face, and the arcs represent the distances between different features. Ranking of constellations is based on a probability density function that a constellation corresponds to a face versus the probability it was generated by an alternative mechanism (i.e., nonface).

All methods mentioned so far will probably track faces, but still some issue like locating faces of various poses in complex background is truly difficult. To reduce this difficulty investigator form a group of facial features in the face-like constellations using more robust modeling approaches such as statistical analysis. Burl et al. [56] make use of statistical shape theory on the features detected from a multi-scale Gaussian derivative filter. A probabilistic model for the spatial arrangement of facial features enables higher detection flexibility. The algorithm is able to handle missing features and problems due to translation, rotation, and scale to a certain extent and a success rate of 84 percent accurate detection out of 150 images taken from a lab-scene sequence, is obtained.

The shape statistics are a joint probability density function over $\mathrm{N}$ feature points, represented by $\mathrm{x}_{\mathrm{i}} \mathrm{x} \mathrm{Yi}$, for the eighth feature under the assumption that the original feature points are positioned in the plane according to a general 2N-dimensional Gaussian distribution. They applied the same maximum-likelihood (ML) method to determine the location of a face. One advantage of these methods is that partially occluded face can be located. However, it is unclear whether these methods can be adapted to detect multiple faces effectively in a scene. In [57] introduction of a face constellation, which enables multi-view face detection and localization, there approach is based on establishing a face 
constellation, which is essentially a reference map indicating how all the other face images in the training set are registered with respect to a reference image. A key feature of this work is the formulation of links to establish a match of a given face with a reference image and to detect and localize faces from arbitrary views at different scales. Thus, face images of different poses can eventually be processed, with each major pose forming a node in the constellation graph as shown in Figure 6. As such, the ability of the face detector to handle pose, expression, and illumination variations only depends on the availability of training face images in the database. Therefore, there is no limit on the type of facial variations that the system can cope with as long as the features used to establish the correspondence are robust to local variations encountered in both images to be matched.

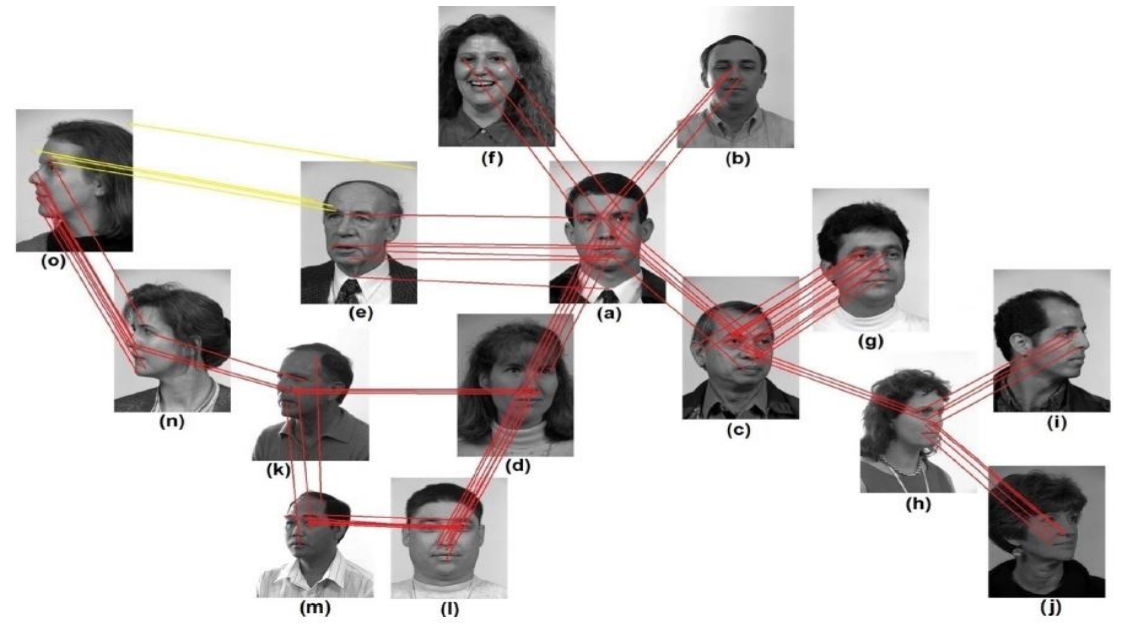

\section{Figure 6. Constellation Formed from Links between Faces of Different Poses and Scales having Reliable Correspondences}

As Shown in Figure 6 a) Reference image selected that has frontal pose and has all the facial characteristics (i.e., no occlusion, defect, etc.). All other images differing in views, from the left profile pose to oblique and right profile poses, are then mapped to the reference map by finding a path that links it to the reference image. Image (l) is registered to the reference map via its link to image (d) and image (a). Such links form the path for registration. As all the correspondences are correct, the link is strong. Image (o) is registered to the reference map through image (e) and image (a). However, there are wrong correspondences between (o) and (e), resulting in a weak link (yellow lines). Such an error will cause wrong registration for image (o) and all images connected to it, such as image (n), the lady's face image in left profile view, if (n) is linked to (a) via the path (o) and (e). Thus, if the weak link can be identified and removed, then (o) can be registered through the path $\{(\mathrm{a}),(\mathrm{d}),(\mathrm{l}),(\mathrm{m}),(\mathrm{k}),(\mathrm{n}),(\mathrm{o})\}$, resulting in correct registration [57].

\section{Face Detection Database}

\begin{tabular}{|c|c|c|c|}
\hline Data Resources & $\begin{array}{c}\text { Number of Images } \\
\text { (Number of } \\
\text { Subjects) }\end{array}$ & Poses & $\begin{array}{c}\text { Original } \\
\text { Image } \\
\text { Size }\end{array}$ \\
\hline & $\begin{array}{c}41368 \text { images } \\
(68 \text { subjects) }\end{array}$ & & \\
& -different poses, & Pan: $0^{\circ}, \pm 22.5^{\circ}, \pm 45^{\circ}, \pm 67.5^{\circ}$, and & $640 \times 480$ \\
& illumination \\
& conditions, and & & \\
& Pixels \\
& & & \\
\hline
\end{tabular}




\begin{tabular}{|c|c|c|c|}
\hline $\begin{array}{c}\text { (2) CMU } \\
\text { Schneiderman's training } \\
\text { database }\end{array}$ & 1506 images & Pan: $0^{\circ} \sim \pm 90^{\circ}$, not estimated & $\begin{array}{c}150 \times 150 \\
\sim \\
659 \times 979\end{array}$ \\
\hline (3) CVL database & $\begin{array}{l}798 \text { images } \\
\text { (114 subjects) } \\
\text {-poses and } \\
\text { expressions }\end{array}$ & Pan: $0^{\circ}, \pm 45^{\circ}$, and $\pm 90^{\circ}$ & $640 \times 480$ \\
\hline $\begin{array}{l}\text { (4) } 04 \text { ' ICPR workshop } \\
\text { benchmark database }\end{array}$ & $\begin{array}{l}1395 \text { images } \\
\text { (15 subjects) }\end{array}$ & $\begin{array}{r}\text { Pan: } 0^{\circ}, \pm 15^{\circ}, \pm 30^{\circ}, \pm 45^{\circ}, \pm 60^{\circ} \\
\quad \pm 75^{\circ}, \text { and } \pm 90^{\circ}\end{array}$ & $384 \times 288$ \\
\hline $\begin{array}{l}\text { (5) BioID Face } \\
\text { Detection Database }\end{array}$ & 1521 images & $\begin{array}{l}\text { varying illumination and complex } \\
\text { background }\end{array}$ & $640 \times 480$ \\
\hline $\begin{array}{c}\text { (6) Non-face background } \\
\text { images }\end{array}$ & 868 images & (None) & $\begin{array}{l}82 \times 54 \sim \\
403 \times 268\end{array}$ \\
\hline $\begin{array}{l}\text { (7) } \mathrm{CMU}+\mathrm{MIT} \text { frontal- } \\
\text { view face test set }\end{array}$ & 130 images & $\begin{array}{c}\text { Frontal view with slight pan or roll } \\
\text { rotations }\end{array}$ & $\begin{array}{l}68 \times 75 \sim \\
1280 \times 10 \\
24\end{array}$ \\
\hline (8) FERET database & 807 images & Pan: $0^{\circ}$ & $256 \times 384$ \\
\hline (9) Yale face database B & $\begin{array}{l}5850 \text { images } \\
(10 \text { subjects })\end{array}$ & Pan: $0^{\circ}, 12^{\circ}$, and $24^{\circ}$ & $640 \times 480$ \\
\hline $\begin{array}{l}\text { (10) CMU Schneider } \\
\text { man's profile test set }\end{array}$ & 208 images & Pan: $0^{\circ} \sim \pm 90^{\circ}$, not estimated & $\begin{array}{l}30 \times 130 \sim \\
456 \times 463\end{array}$ \\
\hline $\begin{array}{l}\text { (11) Face Detection Data } \\
\text { Set and Benchmark } \\
\text { (FDDB) }\end{array}$ & $\begin{array}{c}171 \text { faces in a set of } \\
2845 \text { images }\end{array}$ & Pan: $0^{\circ} \sim \pm 90^{\circ}$, not estimated & $540 \times 480$ \\
\hline
\end{tabular}

\section{Conclusion}

Face detection is currently a very active research area and the technology has come a long way. The last couple of years have shown great advances in algorithms dealing with complex environments such as low quality gray-scale images and cluttered backgrounds. Some of the best algorithms are still too computationally expensive to be applicable or real-time processing, but this is likely to change with coming improvements in computer hardware. This paper presents various numerous feature-based techniques that are available to detect human face. All methods have their own merits and demerits.

\section{References}

[1] S. Borah, S. Konwar, T. Tuithung and R. Rathi, "A Human Face Detection Method Based on Connected Component Analysis", International Conference on Communication and Signal Processing, April 3-5, (2014), India.

[2] I. R. Tsang, J. P. Magalhaes and G. D C. Cavalcanti, "Combined AdaBoost and gradientfaces for face detection under illumination problems", Systems, Man, and Cybernetics (SMC), 2012 IEEE International Conference on, (2012) October 14-17, pp. 2354-2358.

[3] M. A. Berbar, H. M. Kelas and A. A. Kandeel, "Faces and Facial Features Detection in Color Images", Geometric Modeling and Imaging--New Trends, (2006), pp. 209-214.

[4] V. Lekshmi, S. Kumar and D. S. Vidyadharan, "Face Detection and Localization of Facial Features in Still and Video Images", First International Conference on Emerging Trends in Engineering and Technology, (2008), pp. 95-99.

[5] J. Hannuksela, J. Heikkila and M. Pietikainen, "A real-time facial feature based head tracker", Proc. Advanced Concepts for Intelligent Vision Systems, Brussels, Belgium, (2004) , pp. 267-272.

[6] R. Mohamed, M. F. Hashim, P. Saad and S. Yaacob, "Face Recognition using Eigenfaces and Neural Networks", American Journal of Applied Sciences, vol. 2, (2006), pp. 1872-1875. 
[7] J. Chatrath, P. Gupta, P. Ahuja, A. Goel and S. M. Arora, "Real Time Human Face Detection and Tracking", International Conference on Signal Processing and Integrated Networks, Spin, (2014).

[8] J. Yan, X. Zhang, Z. Lei and S. Z. Li, "Face detection by structural models", Image and Vision Computing, vol. 32, no. 10, (2014), pp. 790-799.

[9] H. Pan, Y. Zhu and L. Xia, "Efficient and accurate face detection using heterogeneous feature descriptors and feature selection", Computer Vision and Image Understanding, vol. 117, no. 1, (2013) January, pp. $12-28$.

[10] W.-C. Hu, C.-Y. Yang and D.-Y. Huang, "Feature-based Face Detection against Skin-color Like Backgrounds with Varying Illumination”, Journal of Information Hiding and Multimedia Signal Processing, (2011).

[11] Z. Li, L. Xue and F. Tan, "Face detection in complex background based on skin color features and improved AdaBoost algorithms", Progress in Informatics and Computing (PIC), 2010 IEEE International Conference on, vol. 2, (2010) December 10-12, pp. 723, 727.

[12] E. Hjelmas and B. K. Low, "Face Detection: A Survey," Computer Vision and Image Understanding, vol. 83, (2001) April, pp. 236-274.

[13] G. D. Hager and P. N. Belhumeur, "E_cient region tracking with parametric models of geometry and illumination”, IEEE Transactions on Pattern Analysis and Machine Intelligence, vol. 20, (1998) pp. $1025-1039$.

[14] A. Lanitis, C. J. Taylor, and T. F. Cootes, "\Automatic interpretation and coding of face images using exible models", IEEE Transactions on Pattern Analysis and Machine Intelligence, vol. 19, no. 7, (1997), pp. 742-756.

[15] T. Mcinerney and D. Terzopoulos, "Deformable models in medical image analysis: A survey," Medical Image Analysis, vol. 1, (1996) pp. 91-108.

[16] T. Cootes, C. Taylor, D. Cooper, and J. Graham, "Active shape models-their training and application," Computer Vision and Image Understanding, vol. 61, no. 1, (1995), pp. 38-59.

[17] E. Murphy-Chutorian and M. M. Trivedi, "Head Pose Estimation in Computer Vision: A Survey", IEEE TRANSACTIONS ON PATTERN ANALYSIS AND MACHINE INTELLIGENCE, (2008).

[18] X. Ou, X. Wu, Y. Zhang and Q. Fu,” Mapping Peking Opera Facial Makeup Onto a Human Face Image Based on Facial Landmarks", The 2014 7th International Congress on Image and Signal Processing, (2014).

[19] I. Matthews and S. Baker, “Active appearance models revisited”, Int. J. Comput. Vis. 60 (2004), pp. $135-164$

[20] G. Tzimiropoulos, J. Alabort-iMedina, S. Zafeiriou and M. Pantic, "Generic active appearance models revisited", Proceedings of the Asian Conference on Computer Vision, vol. LNCS 7726, (2013), pp. 650 663.

[21] S. Sangal and A. Bhaumik," A Robust Method For Automatic Emotion Detection From Face Characteristics Active Shape Model Meets Support Vector Machines”, 2014 IEEE International Advance Computing Conference (IACC), (2014).

[22] S. K. Rath and S. S. Rautaray," A Survey on Face Detection and Recognition Techniques in Different Application Domain", I.J. Modern Education and Computer Science, vol. 8, (2014), pp. 34-44

[23] Z. Ghahramani and G. E. Hinton, "The EM Algorithm for Mixtures of Factor Analyzers," Technical Report CRG-TR-96-1, Dept. Computer Science, Univ. of Toronto, (1996).

[24] B. J. Frey, A. Colmenarez, and T. S. Huang, "Mixtures of Local Subspaces for Face Recognition," Proc. IEEE Conf. Computer Vision and Pattern Recognition, (1998), pp. 32-37.

[25] H. Rowley, S. Baluja and T. Kanade, "Neural Network-Based Face Detection", IEEE Trans. Pattern Analysis and Machine Intelligence, vol. 20, no.1, (1998) January.

[26] M. J. Jones and P. Viola, "Fast multi-view face detection", Technical Report TR2003-96, Mitsubishi Electric Research Labs, (2004).

[27] H. Takatsuka, M. Tanaka and M. Okutomi, "Distribution-Based Face Detection using Calibrated Boosted Cascade Classifier", 14th International Conference on Image Analysis and Processing (ICIAP 2007).

[28] K.-K. Sung, "Learning and Example Selection for Object and Pattern Detection," PhD thesis, Massachusetts Inst. of Technology, (1996).

[29] K.-K. Sung and T. Poggio, "Example-Based Learning for View-Based Human Face Detection," IEEE Trans. Pattern Analysis and Machine Intelligence, vol. 20, no. 1, (1998) January, pp. 39-51.

[30] M.-H. Yang, D. J. Kriegman and N. Ahuja, "Detecting Faces in Images: A Survey", IEEE TRANSACTIONS ON PATTERN ANALYSIS AND MACHINE INTELLIGENCE, vol. 24, no. 1, (2002) January.

[31] U. Bakshi and R. Singhal, "A SURVEY ON FACE DETECTION METHODS AND FEATURE EXTRACTION TECHNIQUES OF FACE RECOGNITION”, International Journal of Emerging Trends \& Technology in Computer Science (IJETTCS), vol. 3, no. 3, (2014) May-June.

[32] S. Kr. Singh, D. S. Chauhan, M. Vatsa and R. Singh, "A Robust Skin Color Based Face Detection Algorithm, Tamkang Journal of Science and Engineering, vol. 6, no. 4, (2003), pp. 227-234.

[33] http://en.wikipedia.org/wiki/RGB_color_model 
[34] H. A. Alabbasi and F. Moldoveanu, "Human face detection from images, based on skin color", Proceedings of the 18th International Conference on System Theory, Control and Computing, Sinaia, Romania, (2014) October 17-19.

[35] J. L. Crowley and J. Coutaz, "Vision for Man Machine Interaction," Robotics and Autonomous Systems, vol. 19, (1997), pp. 347-358.

[36] D. Cahi and K. N. Ngan, "Face Segmentation Using Skin-Color Map in Videophone Applications", IEEE Transaction on Circuit and Systems for Video Technology, vol. 9, (1999) pp. 551-564.

[37] M J. T. Rainders, P. J. L. van Beek, B. Sankur, J. C. A. van der Lubbe, "Facial Feature Localization and Adaptation of a Generic Face Model for Model-Based Coding", (1994).

[38] B. K. Low, M. K. Ibrahim, “A Fast and Accurate Algorithm Facial Feature Segmentation”, (1997).

[39] J. Yao and W. K. Cham, "Efficient model-based linear head motion recovery from movies", In: IEEE International Conference on Computer Vision and Pattern Recognition, (2004), pp. 414-421.

[40] M. Tkalc 'ic ${ }^{`}$, A. Odic' and A. Košir, "The impact of weak ground truth and facial expressiveness on affect detection accuracy from time-continuous videos of facial expressions", Information Sciences, vol. 249, (2013), pp. 13-23.

[41] M. Modi and F. Macwan, "Face Detection Approaches: A Survey", International Journal of Innovative Research in Science, Engineering and Technology, vol. 3, no. 4, (2014) April.

[42] T. Sakai, M. Nagao, and T. Kanade, "Computer analysis and classification of photographs of human faces", in Proc. First USA-Japan Computer Conference, (1972), p. 27.

[43] I. Craw, H. Ellis, and J. R. Lishman, "Automatic extraction of face-feature", Pattern Recog. Lett. Feb. (1987), pp. 183-187.

[44] S. Anila and N. Devarajan,"Simple and Fast Face Detection System Basedon Edges", International Journal of Universal Computer Sciences, vol.1, no. 2, (2010).

[45] F. Faux and F. Luthon, "Theory of evidence for face detection and tracking", International Journal of Approximate Reasoning, vol. 53, (2012), pp. 728-746.

[46] S. Jeng, H. Liao, C. Han, M. Chern and Y. Liu, "Facial feature detection using geometrical face model: an efficient approach", Pattern Recogn., vol. 31, (1998), pp. 273-282.

[47] P. Viola and M. Jones, "Rapid object detection was using a boosted cascade of simple features", in Proc. Of CVPR, (2001), pp. 511-518.

[48] H. Hatem, Z. Beiji, R. Majeed, M. Lutf and J. Waleed, "Face Detection and Pose Estimation Based on Evaluating Facial Feature Selection”, International Journal of Hybrid Information Technology, vol. 8, no. 2, (2015), pp. 109-120.

[49] C. P. Papageorgiou, M. Oren, and T. Poggio, “A General Framework for Object Detection”, Proc. IEEE Int'l Conf. Computer Vision, (1998), pp. 555-562.

[50] X. Luo, "ALGORITHMS FOR FACE AND FACIAL EATURE DETECTION", Master of Science Thesis, (2006).

[51] Y. Freund, "An adaptive version of the boost by majority algorithm", in COLT: Proceedings of the Workshop on Computational Learning Theory, Morgan Kaufmann Publishers, (1999).

[52] Y. Freund and R. E. Schapire, "Experiments with a new boosting algorithm", in Proc. International Conference on Machine Learning, (1996), pp. 148-156.

[53] http://en.wikipedia.org/wiki/AdaBoost

[54] C. Zhang and Z. Zhang, "A Survey of Recent Advances in Face Detection", Boosting-Based Face Detection and Adaptation" by Cha Zhang and Zhengyou Zhang, Morgan \& Claypool Publishers, (2010).

[55] O. Tuzel, F. Porikli, and P. Meer, "Region covariance: A fast descriptor for detection and classification", In Proc. Of ECCV, (2006).

[56] M. C. Burl, T. K. Leung, and P. Perona, "Face Localization via Shape Statistics," Proc. First Int'l Workshop Automatic Face and Gesture Recognition, (1995) pp. 154-159.

[57] S. M. H. Anvar, W.-Y. Yau and E. K. Teoh, "Multiview face detection and registration requiring minimal manual intervention", IEEE transactions on pattern analysis and machine intelligence, vol. 35, no. 10, (2013) October.

\section{Authors}

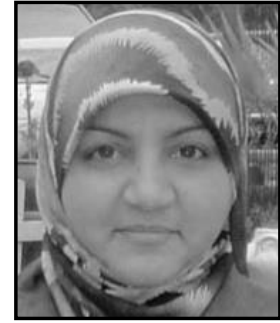

Hiyam Hatem, she received the BS degree from the department of computer science, Baghdad university, Iraq, 2003. The master degree from Huazhong University of science and technology Wuhan, China, in 2010.Currently, she is a PhD student in School of information science and Engineering at Central south university, Changsha, China. Her research interests include face processing and recognition, object detection, pattern recognition, computer vision, and biometrics. 


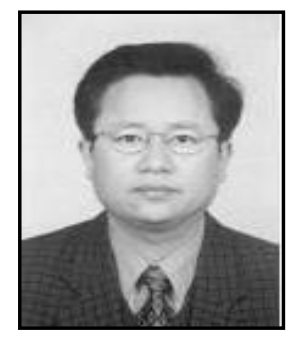

Zou Beiji, he holds a Ph.D. in Computer science and technology from Hunan University, China. He is currently a Professor in School of Information Science and Engineering of Central South University, China. He worked at Zhejiang University, China as a visiting scholar from Jul.1999 to Jun. 2000 and at Tsinghua University, China as a post-doctor from Jan. 2002 to Nov. 2003 respectively, engaged in research about Human Facial Expressional Recognition and Animation. He worked in the research field of Multimedia Technology at Griffith University in Australia from Dec. of 2003 to Dec. of 2004.Interest area of research: Digital Image Processing, Computer Graphics, Multimedia technology, Software Engineering.

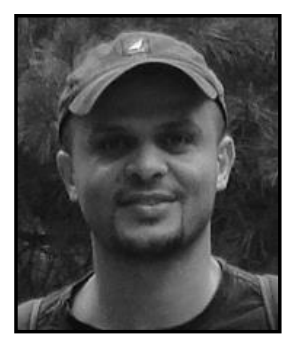

Raed Majeed Muttasher, he received his B.Sc. In Computer Science from Baghdad University, colleges of science, computer department in 2004. Received the Master Degree in applied computer technology from Wuhan University, school of computer in 2011, P.R. China. He is currently working toward his Ph.D. degree at Central South University, School Of Information Science And Engineering, P.R. China. His research interests include 3D Object Recognition, 3D Modeling, Pattern Recognition, and Image Processing. 